\title{
Studying the Law of the Movement of Cotton Particle on a Saw Cylinder and the Interaction with Saw Teeth
}

\section{Jamoliddin Ergashev*, Khamid Akhmedhodjaev, Abdusamat Karimov, Juramirza Kayumov, Rashida Ergasheva, Shohruxmirzo Mahsudov}

Namangan Institute of Engineering and Technology, Namangan, Uzbekistan

Email: *ergashev64@mail.ru

How to cite this paper: Ergashev, J., Akhmedhodjaev, K., Karimov, A., Kayumov, J., Ergasheva, R. and Mahsudov, S. (2019) Studying the Law of the Movement of Cotton Particle on a Saw Cylinder and the Interaction with Saw Teeth. Engineering, 11, 717-725.

https://doi.org/10.4236/eng.2019.1110046

Received: August 17, 2019

Accepted: October 22, 2019

Published: October 25, 2019

Copyright $\odot 2019$ by author(s) and Scientific Research Publishing Inc. This work is licensed under the Creative Commons Attribution International License (CC BY 4.0).

http://creativecommons.org/licenses/by/4.0/

c) (i) Open Access

\begin{abstract}
This article investigates the movement trajectory of the cotton particle from Point D of belt transporter of the saw gin camera to Point B of the saw cylinder. In other words, the forces acting on the cotton particle and the equations of their interactions are developed, where, the differential equation of the law of movement of the cotton particle "belt + saw" is made according to the d'Alembert principle. Arc-BD of cotton particle gives the length of the arc of their fall on the saw teeth. The maximum separation of fibers and the quality of separation of fiber from seeds, as well as the time ( $t$ ) of rapid separation of fiber from seeds, depend on the length of the arc- $\mathrm{BD}$, because the critical length of the arc-BD makes it possible to reduce the multi-layering of the falling cotton particle on the saw teeth. In this scientific research work, patented by the new working camera of the saw gin, the trajectories of the movement of the cotton particles along the arc-DB, as well as the trajectory of the move in the direction of the grate are studied. The results of the study show that the number of fibers increases along the height of the teeth of the saw with one touch. This leads to a decrease in the average stay of seeds in the working chamber of the gin. As a result, the efficiency of the saw gin is increased.
\end{abstract}

\section{Keywords}

Cotton Particle, Belt Conveyor, Saw Cylinder, Grate, Cotton Seeds, Cotton Fiber, Net Surface

\section{Introduction}

It is known that the production of high-quality cotton fiber, necessary for the 
textile industry is of great importance. The main process equipment for the production of high quality cotton fiber is the saw gin machine [1] [2] [3] [4] [5]. During the ginning process, the seeds, which are completely separated from the fiber, begin to accumulate in the central part of the seed chamber. As a result, the density of the seed chamber increases. This leads to the failure of the seed chamber and damage to the fiber and seeds. Much research has been done on this issue and some positive results have been achieved. Including, the work of G.I. Miroshnichenko [6] substantiates the requirement for saw gin feeders and indicates that the flow of raw cotton fed into the gin working chamber must have a high degree of uniformity both in time and in width of the feeder. With an increase in the density of the seed chamber to $550-600 \mathrm{~kg} / \mathrm{m}^{2}$ in the working chamber without an accelerator, due to the significant increase in lateral conditions, the seed chamber stops rotating and the ginning process is completely stopped. G. I. Boldinsky [7] investigated the theoretical foundations of the optimal saw ginning process. As a result of his theoretical studies, it was shown that: raw cotton ginning is more beneficial to carry out at high peripheral speeds of the saw cylinder, not exceeding, however, critical, for which there is a crushing of seeds within the normal range; forced rotation of the raw roller is necessary, which leads to a significant increase in gin productivity with good fiber quality, but its peripheral speed should not exceed half the peripheral speed of the saw cylinder; reducing the content of defects in the fiber should be achieved not by reducing the performance of gin, but by ginning raw cotton with optimal parameters that reduce the compaction of the seed chamber with increased gin performance.

\section{Determining the Movement of Cotton Particles in the DB Zone}

Above mentioned issue has not been fully resolved. The laws of motion of the fly on the ginning drum have been studied in below [8] [9].

$$
\begin{gathered}
P_{0}=m g ; \\
F_{x}=K_{x} V_{x}(t) ; \\
F_{y}=K_{y} V_{y}(t) ;
\end{gathered}
$$

We will compose a differential equation of the law of movement of the cotton particles "grate + saw" according to the d'Alamber principle (Figure 1).

Fixed Cartesian coordinate system-XOY will place on the left end of the grate.

$$
\left\{\begin{array}{l}
m \frac{\mathrm{d} V_{x}}{\mathrm{~d} t}=-F_{x} ; \\
m \frac{\mathrm{d} V_{y}}{\mathrm{~d} y}=-F_{y}+F_{0} ;
\end{array}\right.
$$

Here: $\mathrm{m}-$ mass of the cotton particle;

$K_{X}, K_{Y}$-aerodynamic drag coefficient;

$V_{0}$-motion speed of belt; 


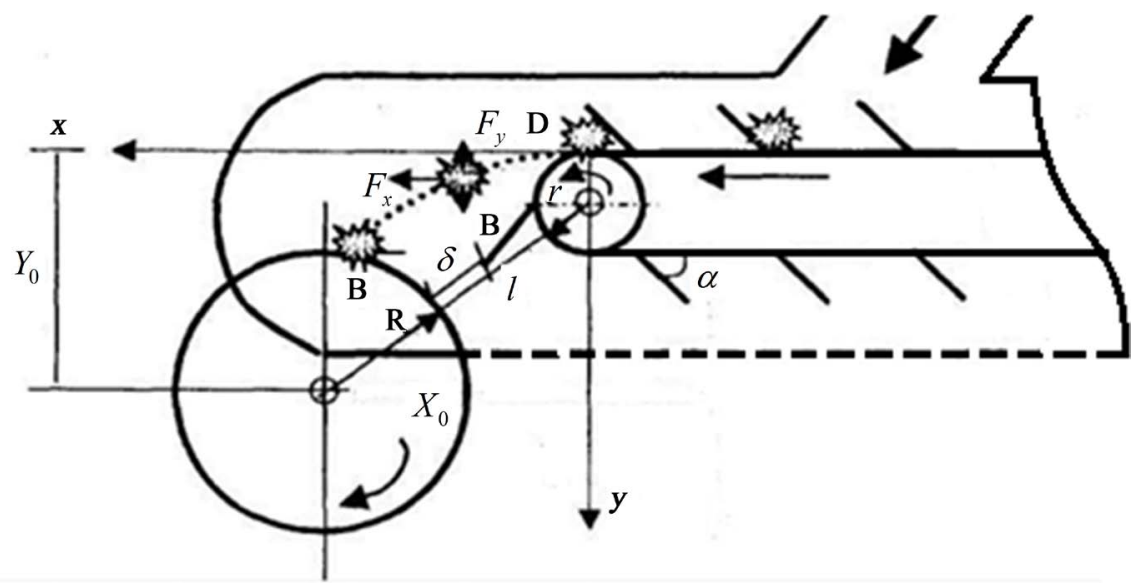

Figure 1. The scheme for determining the movement of cotton particles in the DB zone.

$\alpha$-the angle of the guide bar on the grate;

$F_{X}=F V_{X} ; F_{Y}=K V_{Y}$-aerodynamic drag forces;

$P_{0}=m g-$ Cotton particle gravity;

$V_{X}, V_{Y}$-horizontal and vertical components of the cotton particle speed;

$$
\left\{\begin{array}{c}
\frac{\mathrm{d} V_{x}}{\mathrm{~d} t}=-\omega^{2} V_{x} ; \\
\frac{\mathrm{d} V_{y}}{\mathrm{~d} t}=-\omega^{2} V_{y}+\frac{g}{\omega^{2}} ; \\
\omega^{2}=\frac{k}{m} ;
\end{array}\right.
$$

Having integrated Equation (5), we determine the velocities $V_{X}, V_{Y}$ of the cotton particle on the coordinate axis [10]:

Initial conditions:

$$
\begin{gathered}
\left\{\begin{array}{l}
t=0 ; \\
V_{x}(0)=V_{0} \cos \alpha ; \\
V_{y}(0)=0 ;
\end{array}\right. \\
\left\{\begin{array}{l}
V_{x}=\mathrm{e}^{-\omega^{2} t} C_{1} ; \\
V_{y}=\mathrm{e}^{-\omega^{2} t}+\frac{g}{\omega^{2}} t+C_{2} ;
\end{array}\right.
\end{gathered}
$$

From the initial conditions we find $C_{1}, C_{2}$;

$$
\begin{aligned}
& C_{1}=V_{0} \cos \alpha ; \quad C_{2}=1 \\
& \left\{\begin{array}{l}
V_{x}=V_{0} \cdot \cos \alpha \cdot \mathrm{e}^{-\omega^{2} t} ; \\
V_{y}=\mathrm{e}^{-\omega^{2} t}+\frac{g}{\omega^{2}} t-1 ;
\end{array}\right.
\end{aligned}
$$

From the law of motion:

$$
\left\{\begin{array}{l}
\dot{X}(t)=V_{x} \\
\dot{Y}(t)=V_{y}
\end{array}\right.
$$


We determine the trajectory of the cotton particle by integrating Equation (10).

$$
\left\{\begin{array}{l}
X(t)=-\frac{V_{0} \cos \alpha}{\omega^{2}} \mathrm{e}^{-\omega^{2} t}+\bar{C}_{1} \\
Y(t)=-\frac{1}{\omega^{2}} \mathrm{e}^{-\omega^{2} t}+\frac{1}{2 \omega^{2}} t^{2}-t+\bar{C}_{2}
\end{array}\right.
$$

From the initial conditions: $\mid \begin{aligned} & t=0 ; \\ & X(0)=0 ; \text { the system will have the following } \\ & Y(0)=0 ;\end{aligned}$ expression:

$$
\bar{C}_{1}=\frac{V_{0} \cos \alpha}{\omega^{2}} ; \bar{C}_{2}=\frac{1}{\omega^{2}} ;
$$

So, the trajectory of the cotton particle will be as follows:

$$
\left\{\begin{array}{l}
X(t)=\frac{V_{0} \cos \alpha}{\omega^{2}}\left(1-\mathrm{e}^{-\omega^{2} t}\right) ; \\
Y(t)=\frac{1}{\omega^{2}}\left(1-\mathrm{e}^{-\omega^{2} t}\right)+\frac{q}{2 \omega^{2}} t^{2}-t ;
\end{array}\right.
$$

It is known that in this process the determination of the points of incidence $B$ $\left(X_{1} ; Y_{1}\right)$ of cotton particles on the circumference of the saw is important [11] [12].

Regarding the coordinate system-XOY, the saw circumference equation will have the following form:

$$
\left(X-X_{0}\right)^{2}+\left(Y-Y_{0}\right)^{2}=R^{2}
$$

Here:

$$
\begin{aligned}
& X_{0}=(R+l+r+\delta) \cos \alpha \\
& Y_{0}=(R+l+r+\delta) \sin \alpha
\end{aligned}
$$

$R$-saw radius;

$r$-tape disk radius;

l-length of the guide bar;

$\delta$-distance between saw teeth and guide bar;

$\operatorname{tg} \beta=\frac{Y_{1}}{X_{1}}$-angle of impact of the cotton particle on the saw teeth.

Define the arc BD-distance of the incidence of the cotton particle on the saw teeth. To do this, determine the position of points $B$ and $D$ : if, $\alpha=0$,

$$
\left\{\begin{array}{l}
X(t)=\frac{V_{0}}{\omega^{2}}=\left(1-\mathrm{e}^{-\omega^{2} t}\right) \\
Y(t)=\frac{1}{\omega^{2}}\left(1-\mathrm{e}^{-\omega^{2} t}\right)+\frac{q}{2 \omega^{2}} t^{2}-t \\
\left(X_{1}-X_{2}\right)^{2}+\left(Y_{1}-Y_{2}\right)^{2}=R^{2}
\end{array}\right.
$$

The solution of Equation (17) is given by the coordinates of points $B\left(X_{1} ; Y_{1}\right)$. If $\alpha \neq 0$, 


$$
\left\{\begin{array}{l}
X(t)=\frac{V_{0} \cos \alpha}{\omega^{2}}\left(1-\mathrm{e}^{-\omega^{2} t}\right) \\
Y(t)=\frac{1}{\omega^{2}}\left(1-\mathrm{e}^{-\omega^{2} t}\right)+\frac{q}{2 \omega^{2}} t^{2}-t \\
\left(X_{1}-X_{2}\right)^{2}+\left(Y_{1}-Y_{2}\right)^{2}=R^{2}
\end{array}\right.
$$

The solution to system (18) is given by the coordinates of the points $D\left(X_{2}\right.$; $\left.Y_{2}\right)$.

Arc-BD of cotton particles gives the length of the arc of their incidence on the saw teeth. The maximum separation of cotton particles and the quality of separation of fiber from seeds, as well as the time $(t)$ of rapid separation of fiber from seeds, depends on the length of the arc- $\mathrm{BD}$, because the critical length of the arc-BD makes it possible to reduce the multi-layering of the incidence on the saw teeth.

\section{Study of the Laws of Interaction of the Cotton Particle with the Saw Teeth}

When separating the fibers from the cotton seeds, their interaction with the teeth of the saws is important. It is known that in order to separate the entire fiber from the cotton seeds, it is necessary to repeatedly interact with the teeth of the saw. Below, we study the mathematical model of the laws of interaction of the cotton particle with the saw teeth of the proposed working chamber of the saw fiber separator.

Model of the mathematical and dynamic action of the teeth of a saw with cotton particle, fiber and seeds is shown in Figures 2-4. The forces acting to the cotton particles:

$$
P=m g-\text { cotton particle gravity; }
$$

$F_{m}=C_{x} \cdot X$-the force of attraction of the teeth of the saw acting on cotton fiber;

$C_{X}$ - fiber stretch resistance;

$X$-movement of cotton particle in $X$ axis;

$Y$-movement of cotton particle in $Y$ axis;

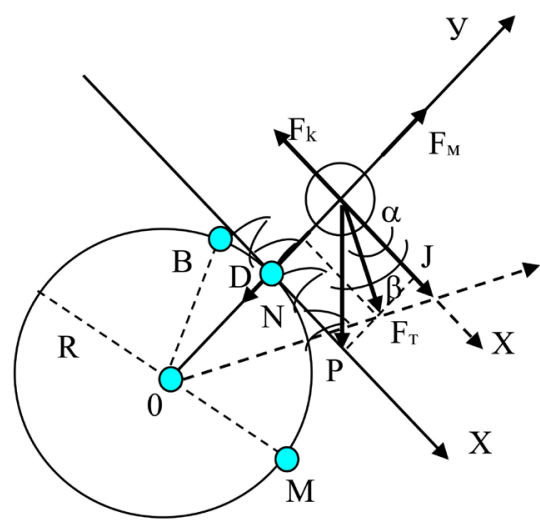

Figure 2. Scheme of the laws of interaction of the cotton particle with the saw teeth. 


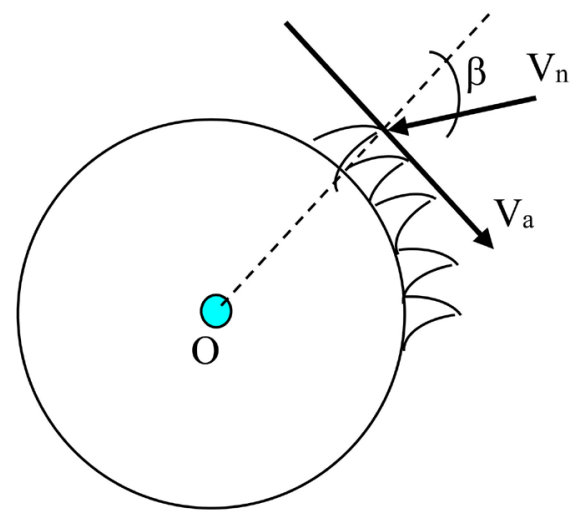

Figure 3. Scheme of the laws of interaction of the cotton fibre with the saw teeth.

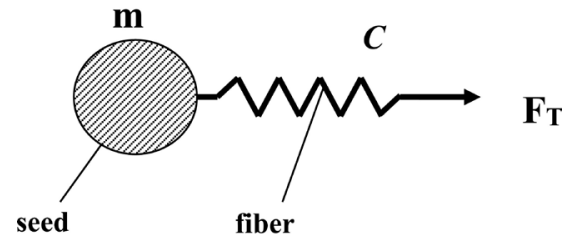

Figure 4. Model of separation of fibers from seeds.

$F_{M}=\frac{m\left(V_{a}-V_{n} \sin \alpha\right)^{2}}{R}-$ centrifugal force acting on cotton particles;

$m$-mass of the cotton particle;

$V_{a}$-Peripheral speed of saw;

$V_{n}$-flying speed of cotton particle;

$I$-inertion force;

$N$-pressure force of cotton particle on saw teeth;

$F_{k}=C_{k}\left(X-V_{a} * t\right)$-inter-friction force of cotton particle;

$X_{0}=V_{a} \cdot t$-movement of the tangential movement of the saw teeth;

$\beta$-Angle of incidence of cotton particle relative to the axis DY.

The differential equation of interaction of the system "cotton particle-saw teeth" based on the d'Alamber principle, relative to the coordinate axis of the $\mathrm{XDY}$ is expressed as follows:

$$
\left\{\begin{array}{l}
m \ddot{x}-F_{k}+F_{T} \cos \alpha+P \cos \beta=0 \\
m \ddot{y}+F_{M}-N+P \sin \beta-F_{T} \sin \alpha=0
\end{array}\right.
$$

Here, $\cos \alpha=\frac{X_{0}}{l_{0}} ; \sin \alpha=\frac{Y_{0}}{l_{0}} ; l_{0}$-the average length of cotton fibers caught in the teeth of the saw;

$X_{0}$-distance between teeth caught in fibers;

$Y_{0}$-teeth height;

$\frac{Y_{0}}{l_{0}}$-taking into account the minimal ratio, we take $\sin \alpha \approx 0$; then, after replacing the expression of all forces, equation system (21) will take the following form: 


$$
\left\{\begin{array}{l}
\ddot{x}+\frac{C_{x}}{m} \cos \alpha x-\frac{C_{k}}{m} x=-g \cos \beta-\frac{C_{k} V_{a}}{m} t \\
\ddot{y}=\frac{N}{m}+g \sin \beta-\frac{V_{a}^{2}}{R}
\end{array}\right.
$$

or,

$$
\left\{\begin{array}{l}
\ddot{x}+\omega^{2} x=+a_{1} t+a_{2} \\
\ddot{y}=b_{1}
\end{array}\right.
$$

Here:

$$
\begin{gathered}
\omega^{2}=\frac{C_{x} \cos \alpha-C_{k}}{m} ; \quad a_{1}=-g \cos \beta ; \quad a_{2}=-\frac{C_{k} V_{a}}{m} \\
b_{1}=\frac{N}{m}+g \sin \beta-\frac{V_{a}^{2}}{R}
\end{gathered}
$$

Equation (23) is a differential equation of the process of the "cotton particle-saw teeth" system.

Integrating it, we find an analytical expression of the laws of motion $X(t)$, $Y(t)$.

a)

$$
\begin{gathered}
\ddot{x}+\omega^{2} x=0 ; \\
x=\sin k t ; \ddot{x}=-k^{2} \sin k t ; \\
-k^{2} \sin k t+\omega^{2} \sin k t=0 ; \quad k= \pm \omega ;
\end{gathered}
$$

Private solution:

$$
x=c_{1} \sin \omega t+c_{2} \cos \omega t
$$

b) $\ddot{x}+\omega^{2} x=a_{1} t+a_{2}$; general solution of the equation:

$$
\begin{aligned}
\bar{x}=A t+B & \rightarrow \omega^{2} A t+\omega^{2} B=a_{1} t+a_{2} ; \\
A & =\frac{a_{1}}{\omega^{2}} ; \quad B=\frac{a_{2}}{\omega^{2}} ; \\
\bar{x} & =\frac{a_{1}}{\omega^{2}} t+\frac{a_{2}}{\omega^{2}} ;
\end{aligned}
$$

c) Taking into account Equations (29) and (32), the general solution of Equation (23) will take the following form:

$$
x=x(t)+\bar{x}(t)=c_{1} \sin \omega t+c_{2} \cos \omega t+\frac{a_{1}}{\omega^{2}} t+\frac{a_{2}}{\omega^{2}}
$$

d)

$$
\begin{gathered}
\ddot{y}=b_{1}, \quad y \rightarrow 0 ; \\
y=b_{1} \frac{t^{2}}{2}+c_{3} t+c_{4}
\end{gathered}
$$

$c_{1}, c_{2}, c_{3}, c_{4}$-being unknown coefficients, are determined from the initial conditions.

Initial conditions:

$$
t=0 ; x(0)=x_{0}=0 ; \dot{x}(0)=V_{n} \cos \beta ;
$$




$$
y(0)=h ; \quad \dot{y}(0)=V_{n} \sin \beta ;
$$

Here: $h-$ Height of the saw gin teeth.

$$
\begin{gathered}
x(0)=c_{2}+\frac{a_{2}}{\omega^{2}}=0 ; \quad c_{2}=-\frac{a_{2}}{\omega^{2}} \\
\dot{x}(0)=\omega c_{1}+\frac{a_{1}}{\omega^{2}}=V_{n} \cos \beta ; \\
c_{1}=\frac{V_{n}}{\omega} \cos \beta-\frac{a_{1}}{\omega^{2}} ; \\
y(0)=c_{4}=h \rightarrow c_{4}=h ; \\
\dot{y}(0)=c_{3}=V_{n} \cos \beta ;
\end{gathered}
$$

Therefore, the interaction of the cotton particles with the saw teeth is determined as follows:

$$
\left\{\begin{array}{l}
x(t)=\left(\frac{V_{n}}{\omega} \cos \beta-\frac{a_{1}}{\omega^{3}}\right) \cdot \sin \omega t+\left(-\frac{a_{2}}{\omega^{2}}\right) \cos \omega t+\frac{a_{1}}{\omega^{2}}+\frac{a_{2}}{\omega^{2}} ; \\
y(t)=b_{1} \frac{t^{2}}{2}+V_{n} \cos \beta t+h ;
\end{array}\right.
$$

\section{Conclusion}

It can be seen from the system that oscillations in the form of a sine wave and a cosine wave together with cotton particles and saw teeth occur because of a complex process. Using the formula (43), we can calculate the maximum value of the force Ft of the pulling saw tooth per fiber bundle. In addition, it is possible to determine the change in pressure force $(\mathrm{N})$ of the cotton particles on the saw teeth in time $(\mathrm{t})$.

\section{Conflicts of Interest}

The authors declare no conflicts of interest regarding the publication of this paper.

\section{References}

[1] Miroshnichenko, G.I., et al. (1980) Equipment and Production Technology of Primary Processing of Cotton. Uqituvchi, Tashkent, 120-140. (In Russian)

[2] Mannopov, A., et al. (2001) Cotton Gin Industry. Tashkent Islamic University, Tashkent. (In Russian)

[3] Sarimsakov, O.Sh., Ergashev, J., Ergashev, Sh. and Kayumov, A. (1991) Working Chamber of the Saw Fiber Separator. Copyright Certificate (Patent) of the SU. No. 1680811. Bulletin of Inventions, Moscow, No. 36.

[4] Ergashev, Sh.T., Sarimsakov, O.Sh., Kurbanov, R.N. and Burnashev, R.Z. (1991) The Working Chamber of the Saw Fiber Separator. Copyright Certificate (Patent) of the SU. No. 1693140. Bulletin of inventions, Moscow, No. 43.

[5] Akhmedzhodzhaev, X., Muradov, R., Sarimsakov, O.S. and Kayumov, A. (1991) Fiber Separator. Copyright Certificate (Patent) of the SU. No. 1622432. Bulletin of in- 
ventions, Moscow, No 3.

[6] Miroshnichenko, G.I. (1972) Fundamentals of Designing Machines for Primary Processing of Cotton. Mechanical Engineering, 335.

[7] Boldinsky, G.I. (1970) Theoretical Foundations of the Optimal Process of Saw Ginning and Defect Formation with It. Moscow.

[8] Tillyaev, M.T., Ergashev, J.S., et al. (1989) Saw Gin. TITLI. Bulletin No. 4412508/21-12-199-No. 9, 122.

[9] Panovka, Y.G. (1971) Introduction to the Theory of Mechanical Vibrations. Moscow. (In Russian)

[10] Lensky, A.N. and Loboda, V.M. (1972) Modeling Contact Interactions of Bodies in the Selection of Impact Systems. Mechanics of Machines, 33-34. (In Russian)

[11] Levkovich, B.A. (1938) Elements of the Theory of Gin. State Publishing House, Tashkent. (In Russian)

[12] Burnashev, R.Z. and Muradov, R. (2001) The Dynamic Problem of the Interaction of Raw Cotton with the Working Bodies of Processing Machines. The Journal Problem of Mechanics, No. 3/4, 55-58. 